\title{
Spectropolarimeter for planetary exploration (SPEX): performance measurements with a prototype
}

\author{
Robert Voors $^{* a}$, Scott G. Moon ${ }^{\mathrm{b}}$, Sandro Hannemann ${ }^{\mathrm{b}}$, Jeroen H. H. Rietjens ${ }^{\mathrm{c}}$, Gerard van Harten ${ }^{\mathrm{d}}$, \\ Frans Snik ${ }^{\mathrm{d}}$, Martijn Smit ${ }^{\mathrm{c}}$, Daphne M. Stam ${ }^{\mathrm{c}}$, Christoph U. Keller ${ }^{\mathrm{d}}$, Erik C. Laan ${ }^{\mathrm{e}}$, Adrianus L. \\ Verlaan $^{\mathrm{e}}$, Willem A. Vliegenthart ${ }^{\mathrm{e}}$, Rik ter Horst ${ }^{\mathrm{f}}$, Ramón Navarro ${ }^{\mathrm{f}}$, Klaas Wielinga ${ }^{\mathrm{g}}$ \\ ${ }^{a}$ Dutch Space B.V. Mendelweg 30, 2333 CS Leiden, The Netherlands \\ b cosine Research B.V., Niles Bohrweg 11, 2333 CA Leiden, The Netherlands \\ ${ }^{\mathrm{c}}$ SRON, Sorbonnelaan 2, 3484 CA, Utrecht, The Netherlands \\ ${ }^{\mathrm{d}}$ Sterrenkundig Instituut Utrecht University, Princetonplein 5, 3584 CC Utrecht, the Netherlands \\ ${ }^{\mathrm{e}} \mathrm{TNO}$ Science and Industry, Stieltjesweg 1, $2628 \mathrm{CK}$ Delft, the Netherlands

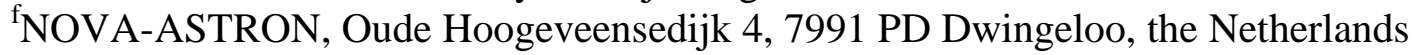 \\ ${ }^{\mathrm{g}}$ Mecon Engineering B.V., Koopmanslaan 25, 7005 BK Doetinchem, the Netherlands
}

\begin{abstract}
SPEX (Spectropolarimeter for Planetary Exploration) was developed in close cooperation between scientific institutes and space technological industries in the Netherlands. It is used for measuring microphysical properties of aerosols and cloud particles in planetary atmospheres. SPEX utilizes a number of novel ideas. The key feature is that full linear spectropolarimetry can be performed without the use of moving parts, using an instrument of approximately 1 liter in volume. This is done by encoding the degree and angle of linear polarization (DoLP and AoLP) of the incoming light in a sinusoidal modulation of the intensity spectrum.

Based on this principle, and after gaining experience from breadboard measurements using the same principle, a fully functional prototype was constructed. The functionality and the performance of the prototype were shown by extensive testing. The simulated results and the laboratory measurements show striking agreement.

SPEX would be a valuable addition to any mission that aims to study the composition and structure of planetary atmospheres, for example, missions to Mars, Venus, Jupiter, Saturn and Titan. In addition, on an Earth-orbiting satellite, SPEX could give unique information on particles in our own atmosphere.
\end{abstract}

Keywords: polarimetry, remote sensing, aerosol, spectropolarimetry, SPEX

\section{INTRODUCTION}

\subsection{An innovative instrument concept}

SPEX (Spectropolarimeter for Planetary EXploration) is an innovative, compact remote-sensing instrument for measuring and characterizing aerosols in the atmosphere. It is capable of accurate full linear spectropolarimetry without moving parts or liquid crystals. High precision polarimetry is performed through encoding the degree and angle of linear polarization of the incoming light in a sinusoidal modulation of the spectrum. Measuring this intensity spectrum thus provides the spectral dependence of the degree and angle of linear polarization. Polarimetry has proven to be an excellent tool to study microphysical properties of atmospheric particles. ${ }^{1}$ Such information is essential to better understand the weather and climate of a planet.

Though SPEX can be used to study any planetary atmosphere, including the Earth's, the current SPEX prototype is tailored to study Martian dust and clouds from an orbiting platform. SPEX' 9 entrance pupils can simultaneously measure intensity spectra from 0.4 to $0.8 \mu \mathrm{m}$, in different directions along the flight direction. This way, the scattering phase functions of dust and cloud particles within a ground pixel are sampled while flying over it. As such, SPEX can

Sensors, Systems, and Next-Generation Satellites XV, edited by Roland Meynart, Steven P. Neeck, Haruhisa Shimoda, Proc. of SPIE Vol. 8176, 81760D · C 2011 SPIE · CCC code: 0277-786X/11/\$18 · doi: 10.1117/12.897706 
provide synergy with instruments on rovers and landers, as it provides an overview of spatial and temporal variations of the Martian atmosphere.

These are some of the key properties of the SPEX prototype instrument

- Compact and low mass

○ 1 liter (spectropolarimeter)

$\circ \quad 2 \mathrm{~kg}$ (including electronics)

○ $2 \mathrm{~W}$ maximum power consumption

- Simultaneous measurement of flux and polarisation state

○ 9 separate fields-of-view

- No moving parts or liquid crystals due to innovative use of birefringent crystals

- Polarisation parameters are encoded in sinusoidal modulation

- Wavelength range 400 to $800 \mathrm{~nm}$

○ Spectral resolution $\sim 2 \mathrm{~nm}$

○ Polarisation resolution $\sim 20 \mathrm{~nm}$

\subsection{Why do we need SPEX?}

Spectropolarimetry is the technique that spectrally resolves polarisation properties of light. Spectropolarimetry can reveal the microphysical properties and spatial/temporal distribution of scattering particles. This in turn can play an important role in the study of the composition and the structure of planetary atmospheres and rings of e.g. Mars, Jupiter, Saturn (Atmosphere, rings, moons) as well as the Earth itself.

SPEX is a very compact and lightweight instrument that has low power consumption that performs spectropolarimetry over an extended field-of-view. This means that it can be put on satellites as well as e.g. in the international space station, while contributing significantly to the science, without putting a large burden on the overall system.

\section{SPECTROPOLARIMETRY}

\subsection{Polarisation modulation}

Spectral polarimetry relies on modulation of the incoming spectrum into different beams of different polarisation state. This can in principle be done in three different ways ${ }^{2}$ :

- Temporal modulation: The observable polarization direction is converted to the fixed linear polarization direction of a regular polarizer by a retarder. This would be a half-wave plate for modulation of Stokes $\mathrm{Q}$ and $\mathrm{U}$, and a quarter-wave plate in case of circular polarimetry (Stokes V). The modulation is obtained by rotating the wave plate. This method has several drawbacks, such as a high risk of failure, high power consumption as well as possible timing issues.

- Spatial modulation: A polarizing beam-splitter divides a beam according to its polarization state, for instance linear polarization at $0^{\circ}$ and at $90^{\circ}$. By simultaneously measuring the intensity of both beams, the polarization degree is obtained. However, spurious signals can be introduced by differential transmissions and aberrations between the two optical systems. This method produces results which are not accurate enough and will lead to a bulky system which is not suitable for space applications.

- Spectral modulation: This spectral modulation is ideally achieved when a sinusoidal modulation of known periodicity is superimposed on the measured spectra for which the amplitude of the modulation scales with the DoLP and the phase of the modulation is determined by the AoLP. A necessary key technology for this method to work are Birefringent crystals.

The spectral modulation is expressed in Equation 1. $O(\lambda)$ is a wavelength dependent offset, $P(\lambda)$ is the degree of linear polarisation (DoLP), $X(\lambda)$ is the angle of linear polarisation (AoLP) and $\delta(\lambda)$ is the spectral retardance.

$$
\frac{I_{s}(\lambda)}{I_{t o t}(\lambda)}=O(\lambda)\left(1 \pm P(\lambda) \cdot \cos \left(\frac{2 \pi \delta(\lambda)}{\lambda}+2 \cdot X(\lambda)\right)\right)
$$




\subsection{The SPEX measurement principle}

The method of spectral modulation is implemented in the SPEX instrument ${ }^{3}$ in a very compact manner and makes use of the following elements, as illustrated in Figure 1.

- Achromatic quarter wave retarder. There are several options for this; for SPEX a Fresnel rhomb was selected due to its robustness (only a single piece of glass) and its large wavelength range. Due to its low refractive index it is impossible to make a Fresnel rhomb out of fused silica, which is the optimal material for use in the UV and has very low intrinsic retardance due to internal stresses. Instead we choose BK7G18, which is spacequalified and radiation resistant.

- A-thermal multiple order retarder with its axes at $45^{\circ}$ from the first retarder. It is made from a crystalline sapphire and $\mathrm{MgF}_{2}$. The optimal thickness ratio of these two for the optical wavelength range is 1:2.4. An overall thickness of $4 \mathrm{~mm}$ of this combination of sapphire and $\mathrm{MgF}_{2}$ has a retardance of $27 \mu \mathrm{m}$, which creates a modulation periodicity of $\sim 25 \mathrm{~nm}$ in the red to $\sim 10 \mathrm{~nm}$ in the blue.

- A Wollaston prism: a polarizing beam splitter aligned with the orientation of the quarter-wave retarder. This can be made from a number of birefringent materials. For SPEX $\alpha$-BBO was selected. Calcite would have been the first choice, but this was shown to be too brittle which would cause failure when mounting the crystals.

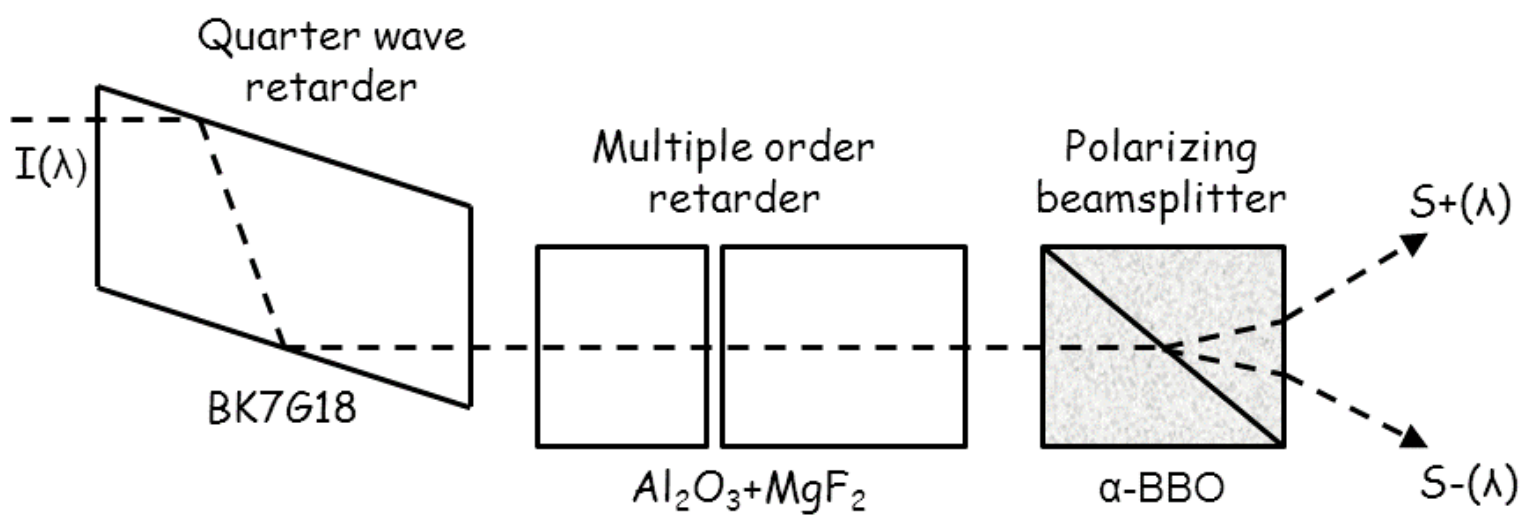

Figure 1 Schematic representation of the polarisation optics used in the SPEX instrument. The incoming light $I(\lambda)$ is spectrally modulated and finally polarized and split into two perpendicular directions, $S+(\lambda)$ and $S-(\lambda)$.

\subsection{The SPEX instrument simulator}

In this paper we describe how the measured data correspond to simulated data. The simulations are performed with the SPEX instrument simulator. The simulator calculates the detected radiation received by the SPEX focal plane array. It simulates the optical and electronic response of SPEX on the incoming spectrum by using the concept of a travelling optical or electronic spectrum. It receives as input a multi-dimensional array of Stokes-vectors, and calculates the effect of the polarizing optics and imaging optics on the spectrum, and finally the detection of this spectrum by the detector and the generation of raw measurement data. The full Stokes-vector is used to account for polarization dependent properties of the optical and dispersive elements.

The conversion of the traveling optical spectrum to a traveling electronic pixel array is performed using interpolation operations. Imaging of the Stokes-spectra in order to resemble the ZEMAX output of the SPEX optical design is performed using (approximate) analytic expressions, and binning on the focal plane array is performed by taking into account the size and position of the focal plane array as well as the pixel size. The detector response and the various electronic noise characteristics are modeled and quantum efficiency, dark current, and read-, shot- and ADC-noise, are included in the calculation. Currently not taken into account are pixel-to-pixel variations in gain and offset, the effects of pixel cross-talk and detector non-linearity, and the contribution of thermal radiation.

Other aspects of the SPEX instrument simulator are:

- Wavelength and temperature dependence of the refractive index of the optical crystals and glasses is modeled

- $\quad$ Absorption in radiation tolerant glasses is modeled using transmission data from Schott. 
- The Fresnel rhomb is modeled using general Mueller matrices for transmission and reflection. The quarter-wave retardance follows automatically when the proper angles for total internal reflection are used.

- A 2D Gaussian is used to model the slit function. The width of this 2D Gaussian may depend on wavelength. The measured slit function is obtained from an image taken with a HgAr spectral line source.

- The nature of the incident light (spectral shape and spectral polarization profile) can be adjusted.

\section{THE SPEX PROTOTYPE}

The SPEX prototype is based on an optical design that consists of two clearly separated parts: the polarisation pre-optics, which harbours a very novel design and a more or less conventional the spectrometer. The pre-optics is made of 9 separate fields-of-view each of which is split into two orthogonal polarisation states. These fields-of-views are created by making of the so-called 'fingers'; each finger contains the very small optical elements that produce the field-of-view. More details on the optics of the SPEX prototype can be found in Snik et $\mathrm{al}^{3}$.. For this prototype only three of the nine fingers were filled; the central field-of-view and the two other fields-of-view ( $\sim 17$ degrees off-nadir). These are referred to as fingers ' 2 ', ' 5 ' and ' 8 ', where ' 5 ' is the nadir FOV and ' 2 ' and ' 8 ' are the other FOVs. This was considered sufficient to be able to convey any finger dependent effects.

\subsection{The polarisation pre-optics}

The optical principle of the polarisation pre-optics is explained in Section 2.2. The optical realization is shown in Figure 2. Light enters the instrument from the right, where a number of baffles are used to reduce stray light. Light is then reflected internally at right angles in the quarter wave retarder, known as the Fresnel rhomb. The Fresnel rhomb is made from BK7G18 glass. Next, light enters the multiple-order retarder. First through the $\mathrm{Al}_{2} \mathrm{O}_{3}$ (Sapphire), next through the $\mathrm{MgF}_{2}$. Last, a Wollaston prism (made from $\alpha$-BBO) acts a polarizing beam splitter. Both beams are then focused onto the slit by a single lens, rather than by two individual lenses; which increases the manufacturability. Note that contrary to most existing polarizing optics designs, this design does not contain any moving parts.

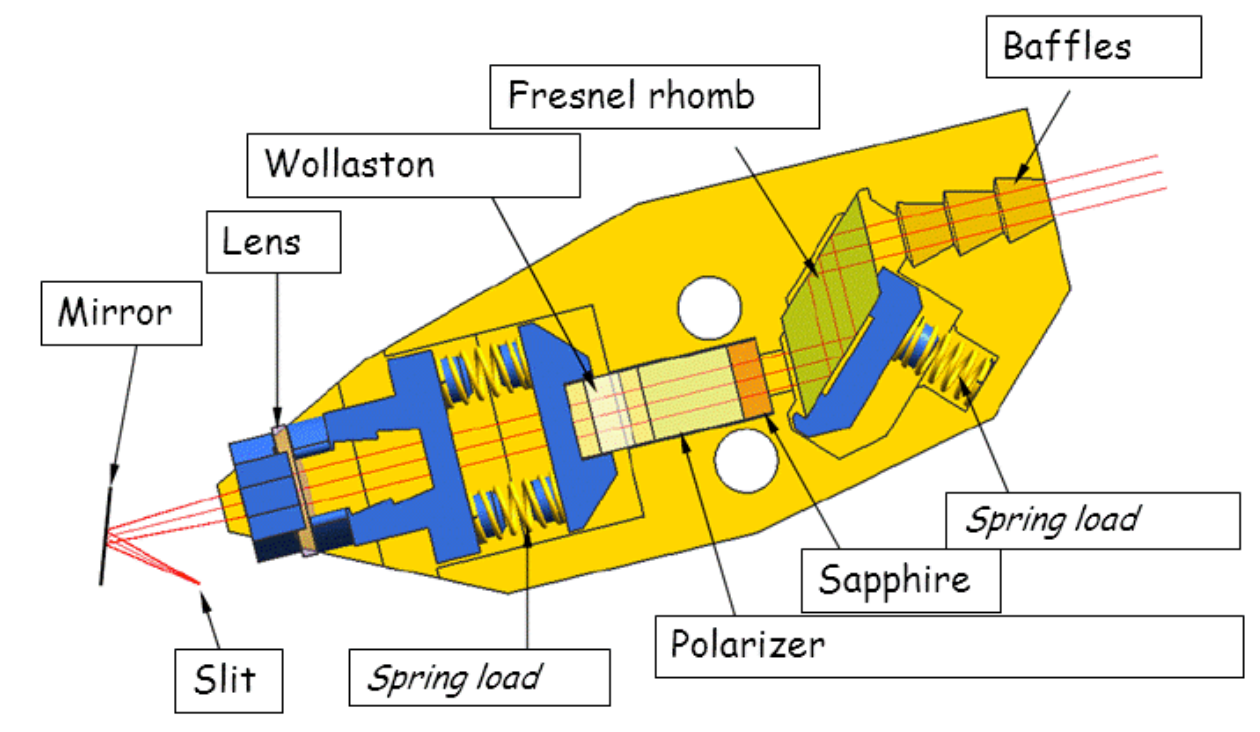

Figure 2 Optical layout of the polarisation pre-optics. 


\subsection{The spectrometer}

The spectrometer disperses the light from the 18 individual beams and images it onto the detector. After the slit, light is first folded by two mirrors. Then it goes through a transmission grating of 750 lines per $\mathrm{mm}$; inherently there will be some overlap of the orders, but the effect of this is limited by the use of the wavelength dependence of the quantum efficiency of the detector that was used. That is, the quantum efficiency of the detector was virtually zero short ward of $400 \mathrm{~nm}$. In addition the longer wavelength region (beyond $750 \mathrm{~nm}$ ) is effectively blocked by a filter.

For this prototype we used an off-the-shelve detector, so design on detector or detector electronics was not done within the scope of the instrument development. The detector used is Retiga 4000R camera from QImaging, which came with all the necessary readout electronics and operating software. It makes use of a Kodak KAI-4021 interline CCD, with approximately $2 \mathrm{kx} 2 \mathrm{k}$ detector pixels. The use of the interline CCD may have had some influence on the measurements and this will not be the type of detector that will be selected for a flight model of the instrument.

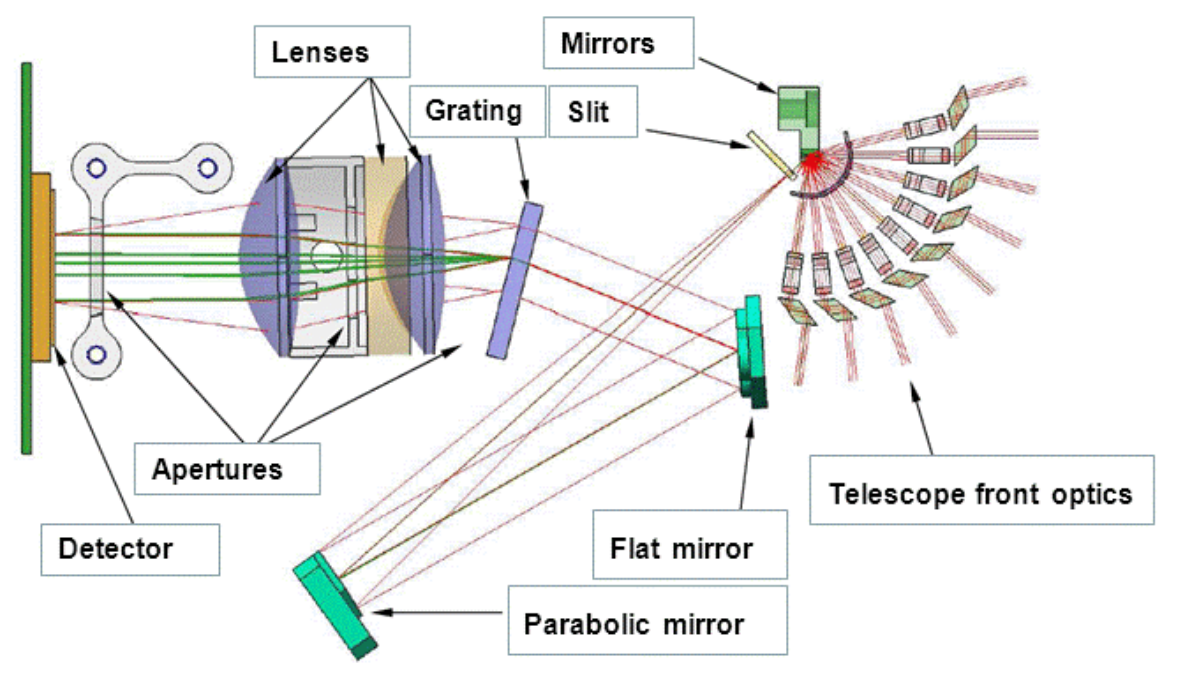

Figure 3 Optical path of the SPEX instrument. Light enters the instrument via 9 different FOVs, is imaged onto the slit and folded through a parabolic and a flat mirror towards the transmission grating. The light then goes through a number of lenses which image onto the detector.

\subsection{Mechanical design}

Complementary to the compact innovative design of the optics, the mechanical design of the SPEX prototype is also compact and robust. Following previous successful optical design like $\mathrm{OMI}^{4}$, SPEX has been designed with a motorblock principle in mind. That is, the main construction - to which the optical elements are fixed - is made from a single block of aluminum. This ensures high temperature and structural stability. The result is a very lightweight (less than $1 \mathrm{~kg}$ ) and compact ( $\sim 1$ liter) opto-mechanical design. FEM modeling has shown that stresses on the optical elements are very low, which should be the case because any stress would induce unwanted bi-refringence in the optical elements. This is achieved by very stiff but low force mounting techniques. Integration of the instrument was facilitated by several smart design techniques, which ensured that no active alignment of the optical elements was necessary for the instrument. 

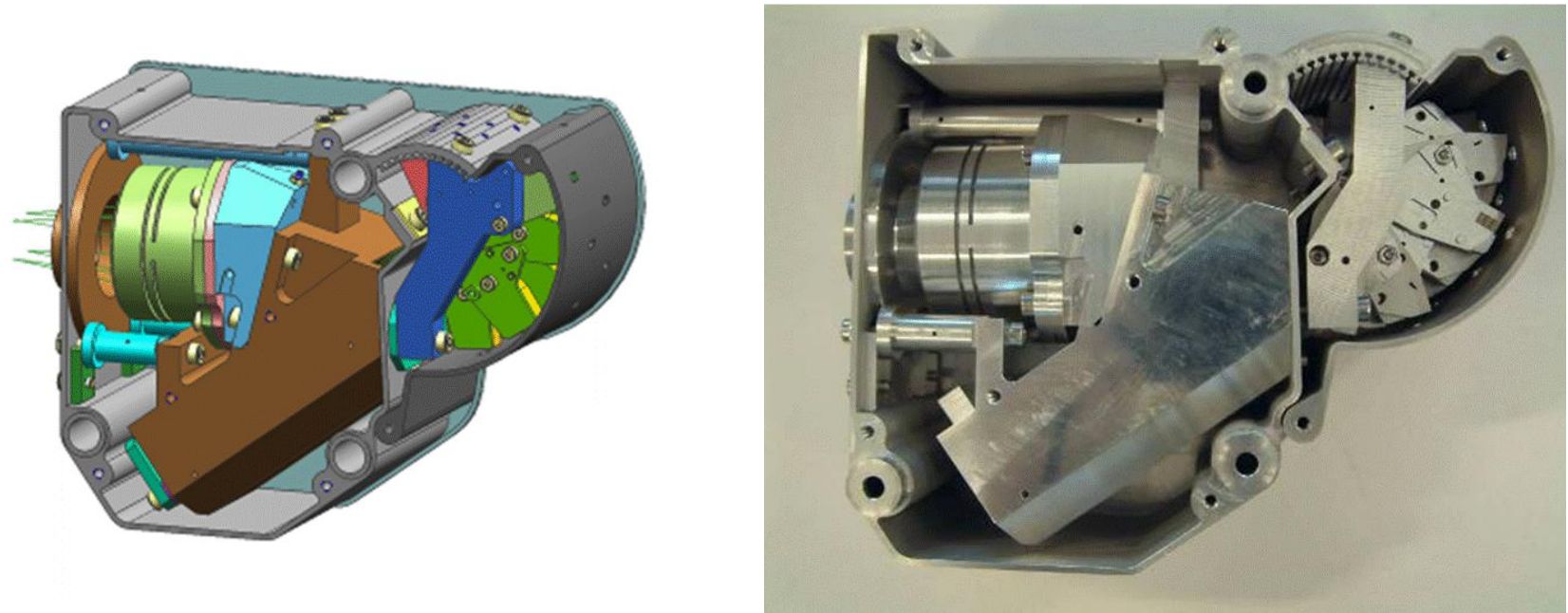

Figure 4 On the left the design of the SPEX prototype is shown and on the right the real instrument, with the cover plate removed

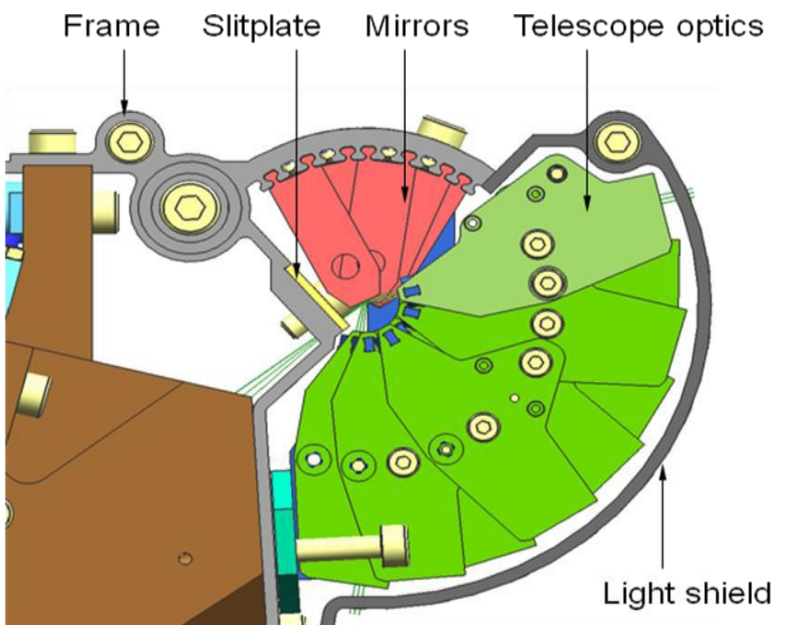

Figure 5 Front optics of the SPEX prototype. The telescope optics (green) contains the optical elements shown in Figure 1

\section{MEASUREMENT RESULTS}

\subsection{Measurement setup}

The SPEX prototype was used in a straightforward optical setup (Figure 6). Two different light sources were available: a white light source and a $\mathrm{HgAr}$ spectral light source. Light from these sources was fed into the optical train by a fiber. Next in the setup is the polarizer, which could be rotated over $180^{\circ}$. It was servo-controlled via Labview. For measurements in which the polarisation angle was changed in small or large steps, after each step, the polarizer was reset and commanded to the new angle from there. This was done in order to maximize repeatability and to prevent any possible hysteresis in the measurements.

In order to limit and position the field-of-view that was offered to the instrument, a circular aperture was placed on an X$\mathrm{Y}$ translation stage. The position parallel to the light beam was not sensitive because it is located in a parallel beam. After the aperture the light is focused onto the entrance of SPEX by a collimator. The SPEX prototype itself was placed 
on a rotation stage, which was stacked on top of a translation stage. This allowed for optimal positioning of the beam w.r.t to the instrument.

The design of the instrument is such that focusing is done as follows; first the detector is screwed onto the instrument and the next step would be to adjust the focus by rotating external screws. However, the detector itself was blocking the screws. Therefore the focus was now accomplished by attaching an extension ring to the camera. The prime criterion for determining the best focus was the sharpness of the fall-off of the edge of the slit image. This meant that first the slit had to be in focus. This was achieved by placing a light source with a strong contrast (visible at the slit) at the end of the lab. After this, a correction was made for the finite distance of the light source.

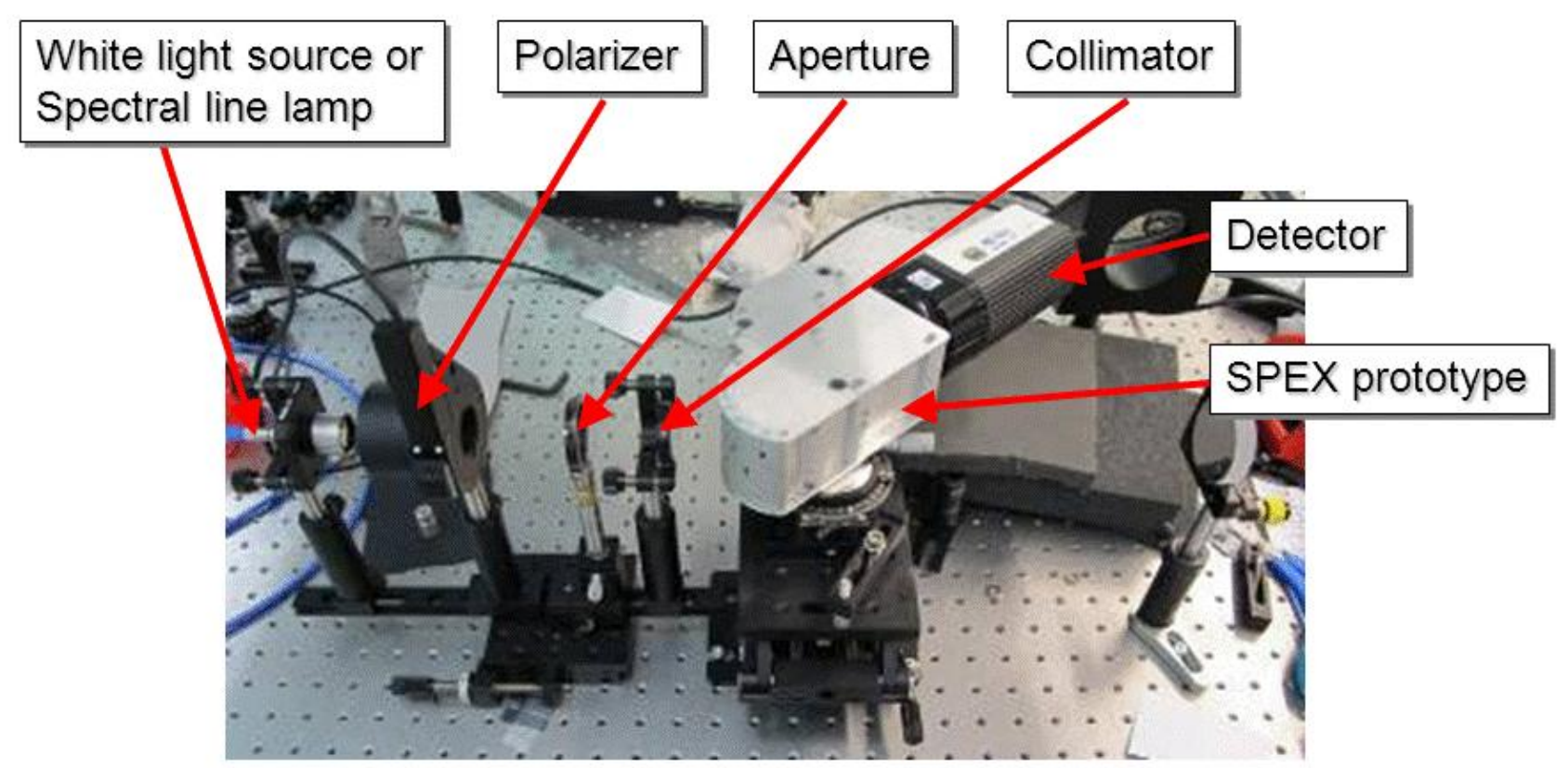

Figure 6 Photograph of the laboratory setup for performance measurements of the SPEX prototype.

\subsection{Spectral calibration}

Spectral calibration of the SPEX breadboard was done by using a $\mathrm{HgAr}$ lamp. Spectral calibration was done with the same setup as the performance measurements, i.e. with the polarizer in the optical train. Lines from the $\operatorname{HgAr}$ lamp are polarized, so in order to obtain a representative calibration, the average of two orthogonal polarizations was taken.

The spectral resolution was measured to be of the right order though slightly larger than expected.

Table 1 Overview of the spectral resolution of the SPEX Instrument for a number of selected wavelengths. These wavelengths are those of some strong emission lines from the $\mathrm{HgAr}$ line lamp.

\begin{tabular}{|l|l|}
\hline Wavelength [nm] & Resolution [nm] \\
\hline 436 & 2.3 \\
\hline 546 & 2.5 \\
\hline 696 & 2.8 \\
\hline 764 & 2.9 \\
\hline 795 & 3.0 \\
\hline
\end{tabular}



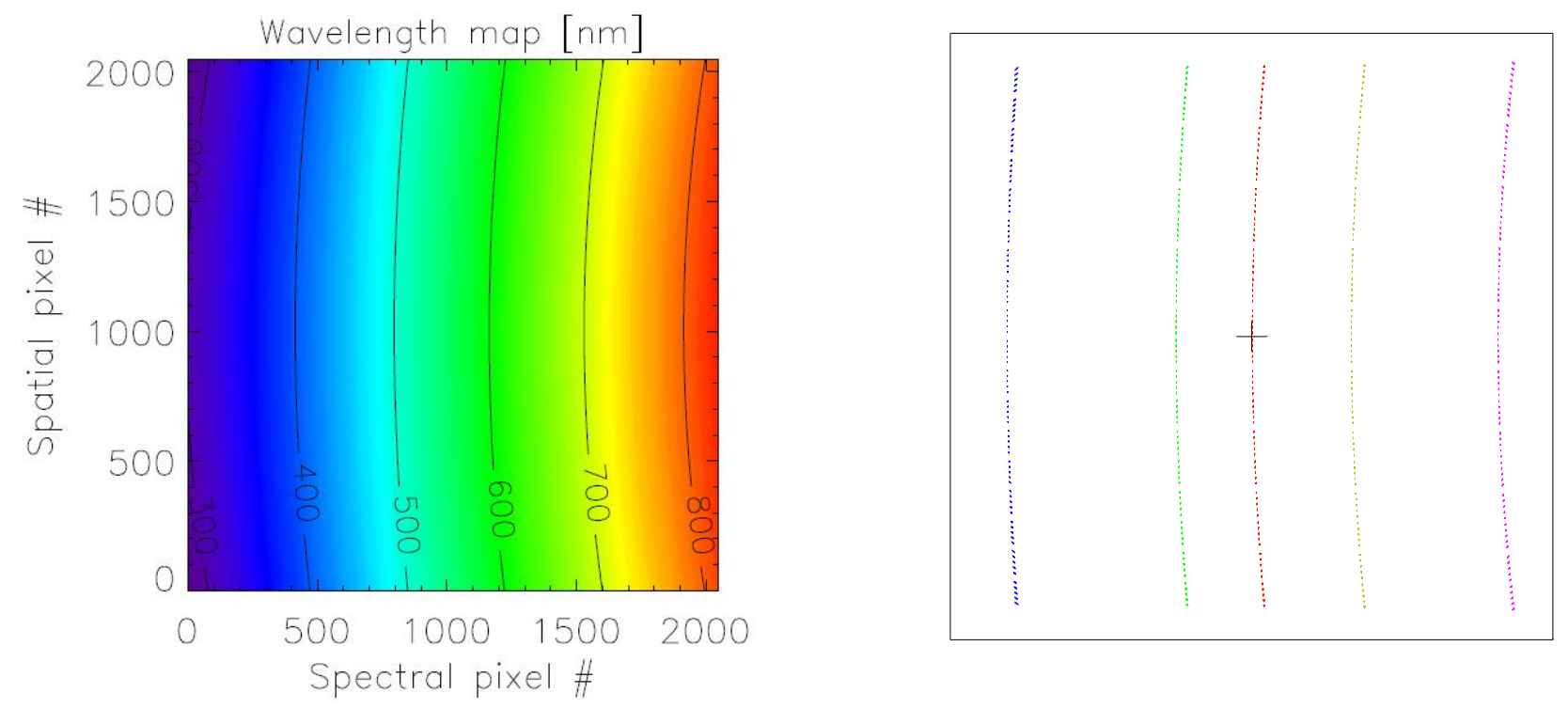

Figure 7: Predicted (left) and measured (right) interpolated wavelength maps. Interpolation between the separate FOVs of the 3 fingers was necessary. The predicted curvature of the field-of-view is well represented by the measurements.

\subsection{Background signal}

It was found there was significant signal in the images at detector areas that are supposed to be dark. This signal was more or less uniform in the spatial direction. A simple test - where the detector was rotated by $45^{\circ}-$ showed that the origin of this spurious signal lies in the electronics. A possible explanation could be light leakage under the masked lines of the interline detector used in the current setup. The spurious signal could be removed very effectively. This correction step also removes the very low dark current in the signal.

In addition to the electronics background signal, stray light was also measured. The total integrated signal in the stray light (Figure 8 ) is between $0.8 \%$ and $1 \%$, for all three fingers. Only in finger 8 there is a hint of a ghost (small local peak) around row 1800 (Figure 8).

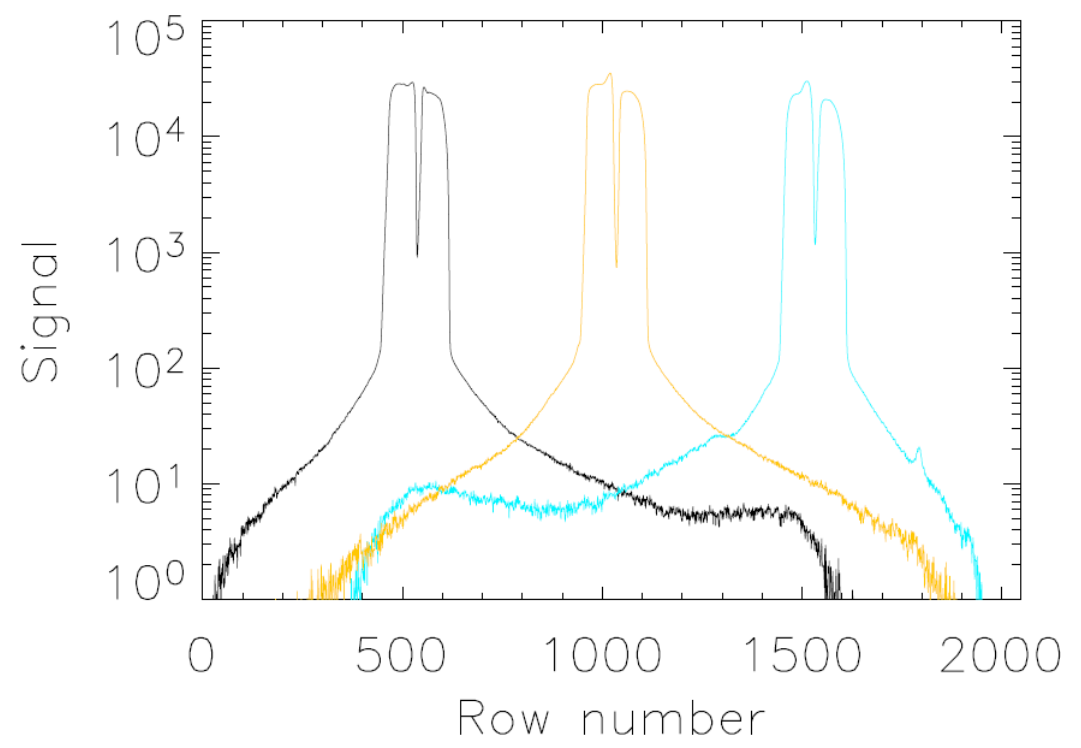

Figure 8 Stray light profiles for the three fingers of the SPEX prototype 


\section{DEMODULATION RESULTS}

Using the SPEX instrument simulator the measurements were modeled according to Eq.1. First, the retardance $\delta(\lambda)$ is determined by fitting a certain spectrum (the reference spectrum) by fixing the angle $X(\lambda)$ to a value independent of wavelength and equal to the AoLP set by the experiment. This reference retardance will be referred to as $\delta_{\text {ref }}(\lambda)$. In this section a total of 4 measurement series are analyzed, each requiring the determination of a reference spectral retardance. These reference retardances are obtained with a demodulation procedure described below and are plotted in Figure 9, together with a calculated spectral retardance using literature values for the refractive index. This 'literature'-retardance is used as an initial guess for the fit routine. The most important observation from this plot is the very good agreement between the spectral retardances of each finger. This should be the case since the small retarder crystals of each finger are produced from the same (larger) crystal plate. The larger deviation around $800 \mathrm{~nm}$ is likely the result of the smaller modulation period and an edge effect.

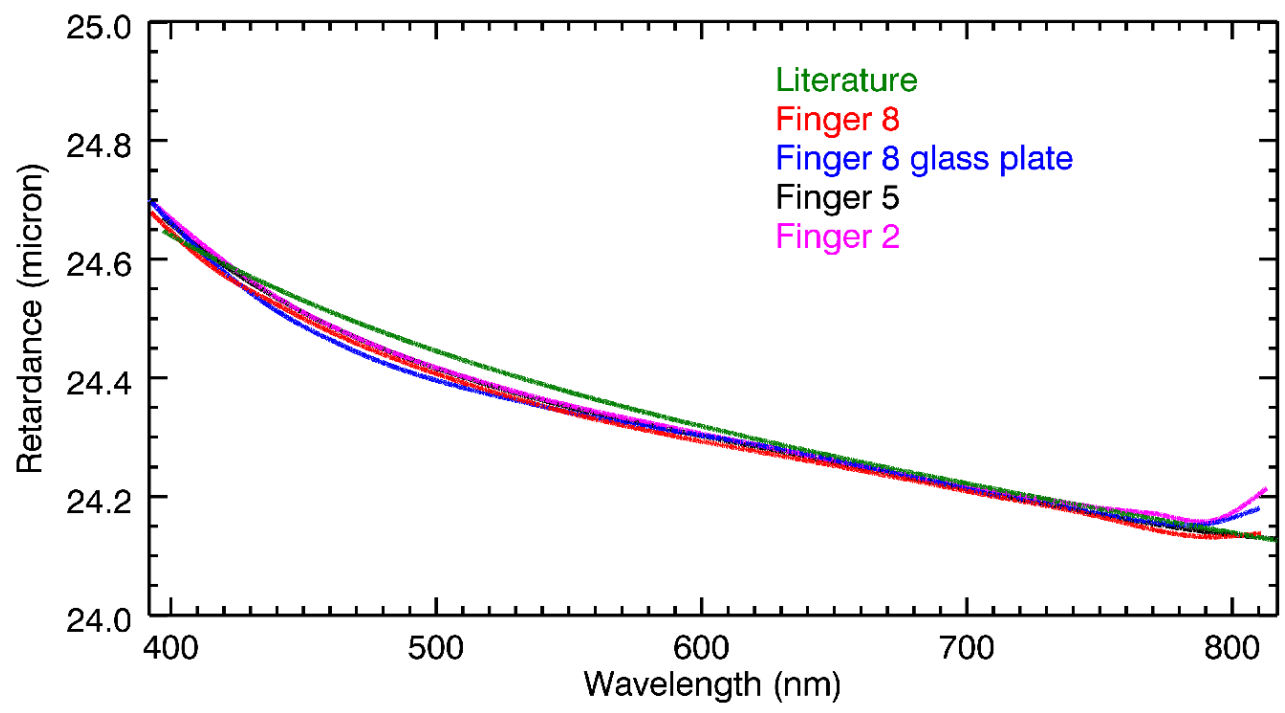

Figure 9 Measured retardance from the three fingers of the SPEX prototype and overplotted the predicted values.

Figure 9 shows that the maximum deviation between the calculated and measured spectral retardance is smaller than $0.3 \%$ over the full spectral range. The agreement for Finger 5 is even better than $0.1 \%$. The offset $O(\lambda)$ and DoLP $P(\lambda)$ are also obtained for this spectrum. Next, all other measurement spectra are fitted by fixing $\delta(\lambda)$ (using $\delta_{\text {ref }}(\lambda)$ from the fit of the reference spectrum) and using a single free fit parameter for obtaining AoLP $X(\lambda)$. Naturally, $X(\lambda)$ is in this case independent of wavelength, which is also expected for the measured spectra.

\section{Fully polarized input}

The first measurements were performed at maximum linear polarisation. The linear polarizer produced high polarisation between $450 \mathrm{~nm}$ and $750 \mathrm{~nm}$. Shortward of $450 \mathrm{~nm}$ the efficiency of polarizer dropped to $\sim 60 \%$ and longward of $750 \mathrm{~nm}$ it dropped to about $40 \%$ at $800 \mathrm{~nm}$. Using the demodulation results of the measurements, the data from the manufacturer were very well modeled.

The angle of the linear polarisation was also very well reproduced, as shown in Figure 10. Two sets of measurement are shown; on the left the full scan over $180^{\circ}$ polarisation angle, in steps of $15^{\circ}$ is shown and on the right, the (arbitrary) range between $45^{\circ}$ and $68^{\circ}$, in steps of $1^{\circ}$. In both cases we see that the input angle is well modeled by the demodulated measurements, to within a few tenths of degrees. It is interesting to note that all three fingers follow a similar pattern, both for the coarse steps and for the fine steps. 

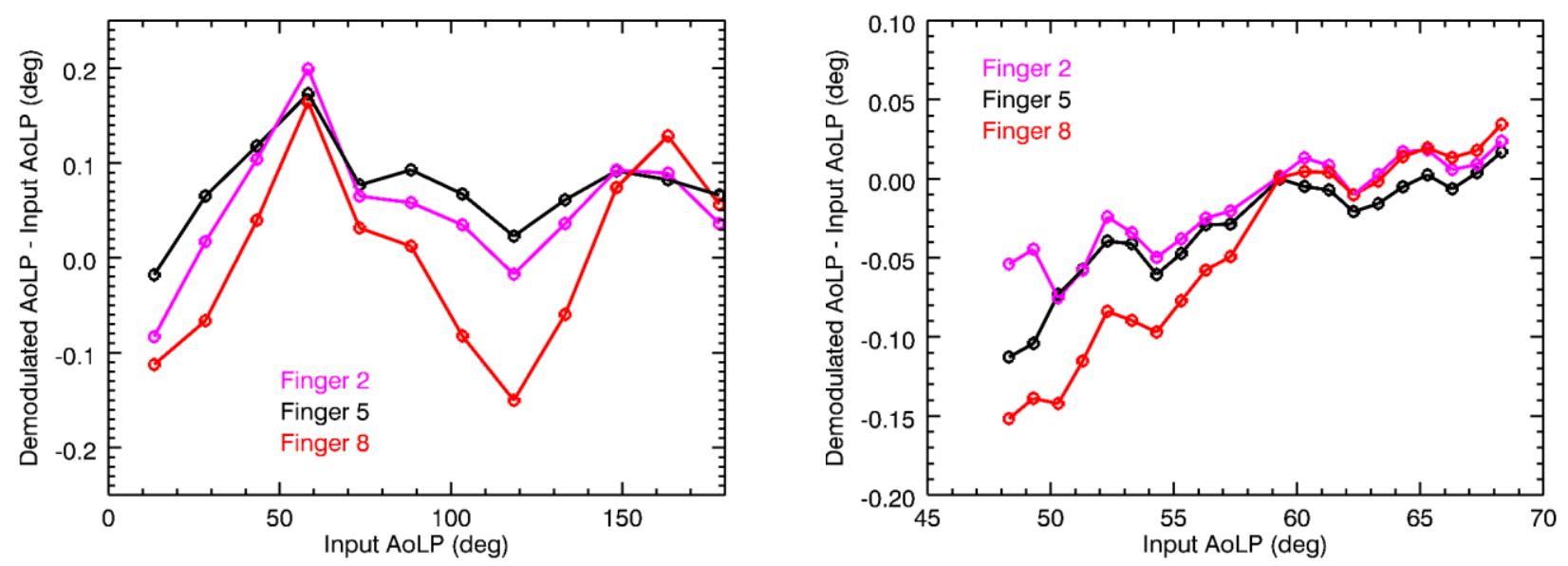

Figure 10 Demodulation results for both the full 180 degrees scan in large steps (left) and a scan over a shorter region with smaller steps (right). The difference between input and modeled AoLP is given for all three fingers.

\section{Glass plate measurements}

In order to produce partially polarized light, in addition to the measurements with the linear polarizer, we made number of measurements with a parallel glass plate in transmission. The same approach as for the linear polarizer was taken to cover a large range $\left(-10^{\circ}\right.$ to $\left.+60^{\circ}\right)$ in coarse steps $\left(5^{\circ}\right)$ and a narrow region $\left(52^{\circ}\right.$ to $\left.58^{\circ}\right)$ in small $\left(0.5^{\circ}\right)$ steps. The result of the demodulated DoLP for the narrow region is shown in Figure 11. We see that differences in DoLP as lows as 0.1\% can be measured with the SPEX prototype.
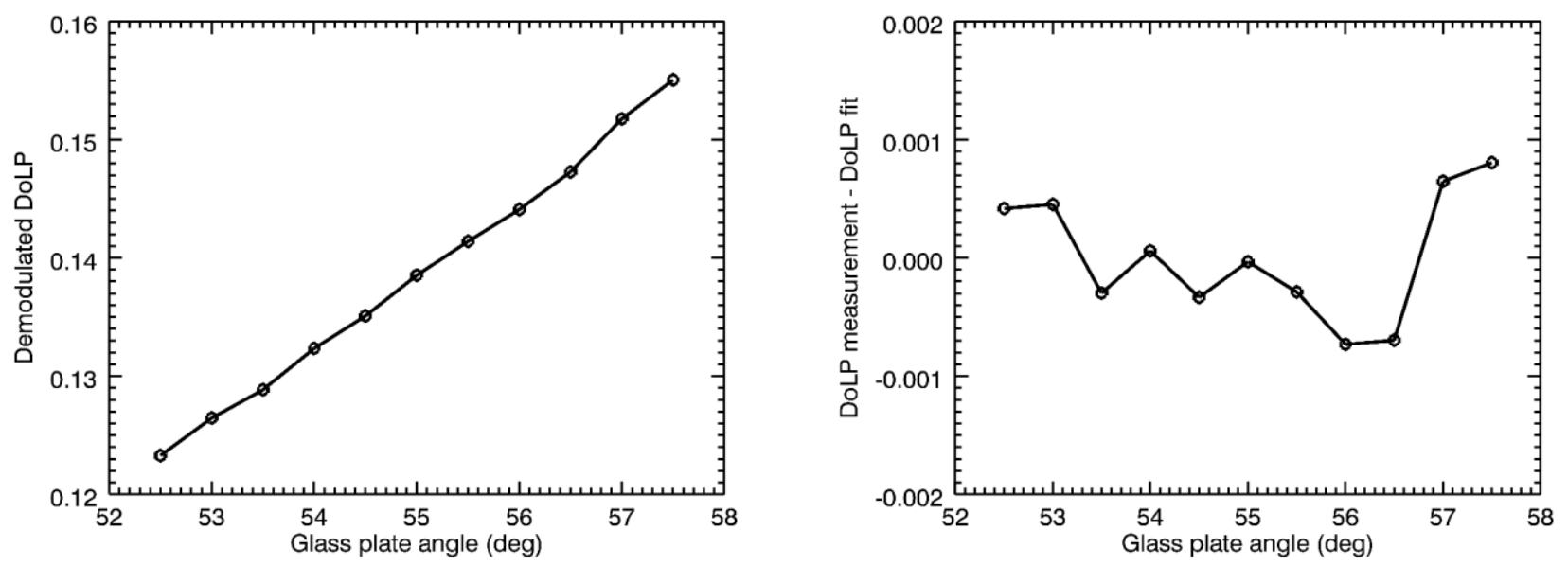

Figure 11 Demodulated DoLP as a function of glass plate angle, obtained from measurement data with Finger 8 illuminated. Right: Difference between demodulated DoLP and a linear fit of the data on the Left.

For the full range covered in coarse steps the results are shown in Figure 12. In order to compare the demodulated DoLP and AoLP with theory, the Mueller matrix of the glass plate for different orientations (e.g. different angle of incidence) has been calculated using the refractive index of BK7 obtained from Schott. Using this Mueller matrix, the DoLP and AoLP can be calculated for each glass plate angle and for different polarisation states of the incident light. 

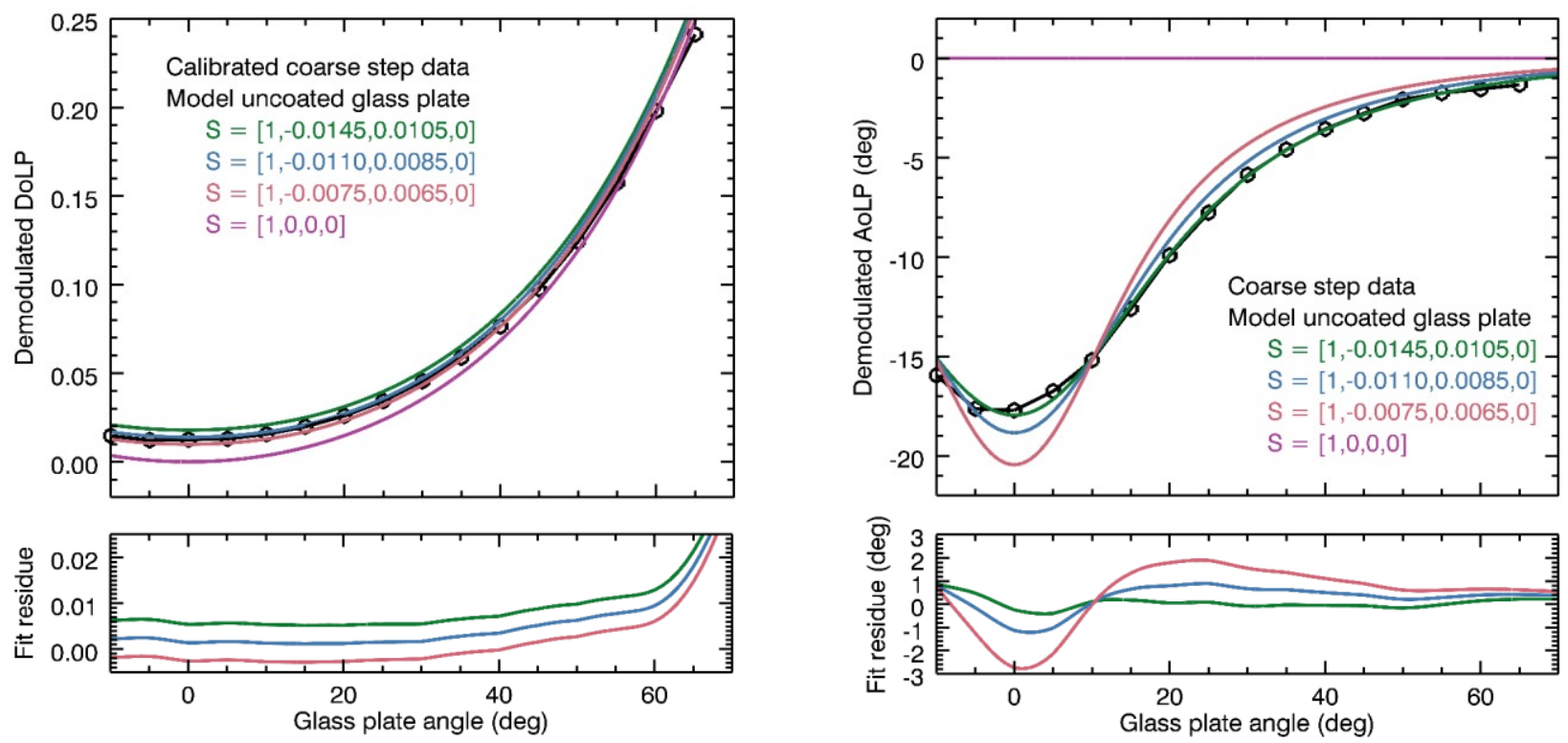

Figure 12: On the top left the calibrated demodulated DoLP is shown as a function of glass plate angle (black) shown together with model calculations using uncoated BK7 and input Stokes vectors [1,0,0,0] (red) and three fit results with weights dominated by the DoLP (magenta), with equal weights (blue) and with weights dominated by the AoLP (green). Also shown are the fit residues (bottom left). On the right the demodulated AoLP are shown as a function of glass plate angle, with the same model calculations as for the left. Also shown are the fit residues.

First, the demodulated DoLP is 'calibrated' using the 'SPEX response function for the DoLP'. Next, a 'look-up table' is produced containing the calculated DoLP and AoLP for each glass plate angle and for different polarisation states of the incident light. The range for both $\mathrm{Q}$ and $\mathrm{U}$ are $-0.015-0.015$ in steps of 0.005 . Finally, using a least-squares minimization algorithm, the best fit of the model data to the both the DoLP and AoLP are calculated, thereby using different weights for the DoLP and AoLP. In Figure 12 both the calibrated DoLP as well as the fitted DoLP are plotted as a function of the glass plate angle $\alpha_{\text {glass }}$ for four input Stokes vectors. Figure 12 shows the demodulated AoLP as well as the fitted AoLP as a function of $\alpha_{\text {glass }}$ for the same four Stokes vectors. These Stokes vectors represent unpolarized light (magenta), the fit result with the largest weight on the DoLP data (red), with roughly equal weights (blue), and with the largest weight on the AoLP data (green).

Using a Stokes vector describing unpolarized light (red curve) yields a DoLP that clearly does not match the data, in particular near $\alpha_{\text {glass }}=0^{\circ}$, as expected. Also, a constant AoLP is observed, which is equal to $0^{\circ}$ because the orientation of rotation axis of the glass plate set by the model is vertical. In order to best match the demodulated AoLP, an incident Stokes-vector $[1,-0.0145,0.0105,0]$ is needed, with a degree of polarisation of $1.8 \%$. However, this incident Stokesvector leads to an overestimation of the DoLP, which is less than $1 \%$ for glass plate angles below $50^{\circ}$. In contrast, the best match to the demodulated DoLP, with an incident Stokes-vector $[1,-0.0075,0.0065,0]$ is needed, with a degree of polarisation of $1.0 \%$, leads to significant deviations of the AoLP.

This resulting difference between the fit and the measurement in the DoLP profile can be explained by one of the following:

- The calibration of the DoLP data is not optimal, leading to an error in the accuracy of about 0.01.

- The lens between the glass plate and SPEX might change the polarisation state of the light emerging from the glass plate.

- The model describing the glass plate uses different optical parameters than apply to the real glass plate.

- Multiple reflections are not modeled. 


\section{CONCLUSIONS AND FUTURE OUTLOOK}

This paper describes the SPEX instrument and explains some of the details behind the theory of spectral modulation as well as an explanation of how this is implemented in a very compact and lightweight design without moving parts. It shows that we have been successful at building this instrument and it highlights the first performance measurements that were performed with this prototype instrument.

The measurements show that with the SPEX instrument it is possible to measure both the degree and the angle of linear polarisation to a degree of accuracy that is required by the scientific community. Full calibration of the instrument still has to be performed, as well as a proper validation of the preliminary results shown in this paper. To do this, on-ground as well as airborne aerosol measurements are planned.

The current SPEX prototype was designed for Mars orbiting mission. A recent opportunity has emerged from CAST (Chinese Academy for Science and Technology) who have offered a flight opportunity on the 2015 Mars orbiter mission HX-1. In addition several other opportunities exist, for which the current SPEX concept can be adapted. One of these opportunities is the study of the icy moons of Jupiter, for which there is currently a feasibility study under way in the Netherlands. Also, SPEX would be ideally suited to observe our own Earth from the ISS platform. ESA recently issued an announcement of opportunity for the study of the Earth's climate for the ISS. A proposal in response to this AO will be issued at the end of 2011 .

\section{ACKNOWLEDGEMENTS}

The design study for SPEX, the prototype construction and testing are supported by PEP grants from the Netherlands Space Office.

\section{REFERENCES}

[1] Hansen, J. E. and Travis, L. D., Space Science Reviews 16, pp. 527-610 (1974)

[2] Snik, F., Karalidi, T. and Keller, C.U., "Spectral modulation for full linear polarimetry", Applied Optics 48, 1337-1346 (2009)

[3] Snik, F., Rietjens, J. H. H., Van Harten, G., Stam, D. M., Keller, C. U., Smit, J. M., Laan, E. C., Verlaan, A. L., Ter Horst, R., Navarra, R., Wielinga, K., Moon, S. G. and Voors, R., "SPEX: The Spectropolarimeter for Planetary EXploration", Proc. SPIE 7731, 77311B (2010)

[4] Levelt, P.F., G.H.J. van den Oord, M.R. Dobber, A. Mälkki, H. Visser, J. de Vries, P. Stammes, J. Lundell and H. Saari, IEEE Trans. Geo. Rem. Sens., Vol. 44, No. 5, 1093-1101, 2006 